Didáctica. Lengua y literatura

ISSN-e: 1988-2548

\title{
La ortografía en el ámbito universitario: una realidad que exige la reflexión del alumnado y la implicación del profesorado
}

\author{
Sergio Suárez Ramírez; Ángel Suárez Muñoz²; Inmaculada Guisado Sánchez³; Miriam \\ Suárez Ramírez ${ }^{4}$
}

Fecha de recepción: 11-10-2017 / Fecha de aceptación: 19-03-2019

Resumen. Hasta hace poco tiempo hemos creído que los errores ortográficos en los que incurrían los estudiantes estaban circunscritos a los niveles educativos no universitarios. Pero cada vez es más evidente que también están presentes entre el alumnado que, se supone, debería tenerlos superados.

Los escritos de diversa índole que produce el alumnado universitario (trabajos académicos, exámenes, consultas o tutorías online, etc.) están plagados de faltas de ortografía que atentan contra el dominio de la competencia en comunicación lingüística e introducen una tarea más para el profesorado encargado de su formación, que hasta ahora habíamos eludido y que ya no justifica, siquiera, atribuirlos a la responsabilidad del profesorado de las etapas educativas previas a la Universidad.

Aun siendo grave esta panorámica, lo es más cuando analizamos la escritura del alumnado universitario de los Grados de Maestro (Infantil y Primaria), que se "forma" para dedicarse a la enseñanza y a la transmisión de contenidos educativos a niños y jóvenes (entre 6 y 16 años).

Como profesores universitarios que padecemos esta realidad queremos transmitir la necesidad, cada vez más imperiosa, de abordar el asunto en las aulas universitarias. El estudio llevado a cabo confirma que no es solo el desconocimiento, sino la falta de atención e interés que muestran los universitarios a la hora de aplicar las reglas ortográficas en sus escritos, las causas principales de tantos errores.

Por eso, creemos que nuestra actuación comienza analizando y describiendo los errores ortográficos de nuestros alumnos para hacerles reflexionar sobre ellos y propiciar una escritura académica correcta. Palabras clave: Ortografía, Universidad, Jóvenes, Investigación, Educación.

1 Departamento de Didáctica de la Lengua

Facultad de Educación. Campus de Soria

Universidad de Valladolid

sergio.suarez@uva.es

2 Departamento de Didáctica de las Ciencias Sociales, de las Lenguas y las Literaturas

Facultad de Educación

Universidad de Extremadura

asuarez@unex.es

3 Departamento de Didáctica de las Ciencias Sociales, de las Lenguas y las Literaturas

Facultad de Educación

Universidad de Extremadura

inguisado@alumnos.unex.es

4 Departamento de Didáctica de las Ciencias Sociales, de las Lenguas y las Literaturas

Facultad de Educación

Universidad de Extremadura

misuarez@unex.es 


\title{
[en] Spelling in the University Education level: a reality that requires the reflection of students and the involvement of teachers
}

\begin{abstract}
Until recently we have considered that spelling by students could only be found at preuniversity educational levels. But it is increasingly evident that they are also present among students in University level, who should have overcome them.

Different writings produced by university students (academic papers, exams, online consultations or tutorials, etc.) have a lot of spelling mistakes that are a serious inconvenient in the acquisition of the linguistic communication competence. Therefore, evaluating spelling mistakes is an additional task for teachers, as guides in education process, which until present days had been considered and exclusive responsibility of teachers at the pre-university educational stages.

Although this panorama is worrying, it is even more so when we analyze the writing of the university students of the Education's degrees (Preschool and Primary Education), who have to learn about how to teach and transmit educational content to children and young people (between 6 and 16 years).

It is important that university teachers make practices and training about spelling in their classrooms, as an urgent issue. The present study confirms that ignorance is not the sole culprit: students do not pay attention and are not interested in this aspect of Grammar when they applying orthographic rules in their writings.

For that it is important to analyze and to describe the main mistakes of our students made as examples in the way to propose a correct academic writing and spelling.
\end{abstract}

Keywords: Spelling, University, Youth students, Research, Education.

\section{[fr] Orthographe dans le domaine universitaire: une réalité qui exige la réflexion des étudiants et la participation des enseignants}

Resumé. Jusqu'à récemment, nous croyions que les fautes d'orthographe encourues par les étudiants étaient circonscrites aux niveaux d'éducation non universitaires. Mais il est de plus en plus évident qu'ils sont également présents parmi les étudiants, qui sont supposés les avoir surmontés.

Les écrits de divers types produits par les étudiants universitaires (travaux universitaires, examens, consultations en ligne ou tutoriels, etc.) sont entachés par des fautes d'orthographe qui minent la compétence linguistique et introduisent une tâche supplémentaire pour le personnel enseignant de leur formation qui jusqu'ici avait échappé et qui ne justifie plus même de les attribuer à la responsabilité des enseignants des étapes de l'enseignement préuniversitaire.

Bien que cette situation semble déjà trés sérieux, elle apparaît plus grave encore quand on analyse l'écriture des étudiants de Master (Enfants et Primaires) qui sont «formés» à se consacrer à l'enseignement et à la transmission de contenus éducatifs aux enfants et aux jeunes et 16 ans).

En tant que professeurs d'université qui souffrent de cette réalité, nous voulons exprimer la nécessité de plus en plus urgente d'aborder la question dans les classes universitaires. L'étude réalisée confirme que ce n'est pas seulement l'ignorance mais le manque d'attention et d'intérêt que les étudiants de l'université mettent à l'application des règles orthographiques dans leurs écrits, causes principales de tant d'erreurs.

Par conséquent, nous croyons que notre action commence par l'analyse et la description des fautes d'orthographe de nos étudiants pour les amener à réfléchir et à promouvoir une écriture académique correcte.

Mots-clés: Orthographe, Université, Jeunesse, Recherche, Education.

Sumario: 1. La ortografía en la Universidad; 2. Los aportes de la investigación; 3. Conclusiones y discusión de los resultados; 4. Bibliografía.

Cómo citar: Suárez Ramírez, S.; Suárez Muñoz, Á.; Guisado Sánchez, I.; Suárez Ramírez, M. (2019). La ortografía en el ámbito universitario: una realidad que exige la reflexión del alumnado y la implicación del profesorado, Didáctica. Lengua y literatura, 31, 135-145. 


\section{La ortografía en la universidad}

Parece mentira que iniciemos este artículo recordando lo que Alvar (1979) ya manifestó como queja: los escasos conocimientos que presentaba el alumnado universitario sobre ortografía. A pesar del tiempo transcurrido, siguen teniendo plena vigencia sus palabras: “... en un curso universitario de Lengua Española un 60 por 100 de mis alumnos tuvo faltas de ortografía en el primer examen parcial...”. (p. 225).

También quedan lejos en el tiempo las reflexiones de Medina (1994) cuando, al escribir sobre la enseñanza de la ortografía en la Universidad, destacó lo que veinte años antes ya afirmara Polo (1974: 47): “es la UNIVERSIDAD el máximo exponente -la más honda consciencia- de la palabra cultura (así debería serlo), y somos nosotros, los docentes de ese nivel, los más obligados a tomar una conciencia más radical y plena de la situación planteada". A lo que Medina añadía: "los alumnos llegan a la facultad con una pésima ortografía y salen de ella igual o peor. ¿Qué podrán, entonces, enseñar a sus futuros discentes?" (1994: 76).

El paso del tiempo, como decimos, no ha hecho más que perpetuar una situación. Observamos y comentamos los déficits en escritura que muestra el alumnado universitario en general. Cualquier proceso comunicativo que utilice el mecanismo de la escritura constata los errores formales y gramaticales en que incurre el alumnado. Díaz Perea (2008) cita algunas investigaciones en el dominio ortográfico del español: Vaca (1983, 1997); Camps et. al. (1990); Matteoda y Vázquez (1990); Salgado (1997) y Gomes de Morais y Teberosky (1998). Todas muestran que el aprendizaje de la convencionalidad ortográfica constituye un problema cognitivo para quien aprende y un desafío pedagógico para quienes enseñan.

Según el Marco Común Europeo de Referencia para las Lenguas, "[la] competencia ortográfica supone el conocimiento y la destreza en la percepción y la producción de los símbolos de que se componen los textos escritos". (2002: 114). La adquisición de la escritura implica un proceso largo y complejo, pues involucra múltiples habilidades en forma simultánea (Smith-Lock, Nickels y Mortensen, 2009). La integración de estas habilidades implica una alta demanda para la memoria de trabajo (Berninger et al., 1992). Esta memoria es limitada, ya que depende en gran medida del grado de automatización de las tareas implicadas (Sánchez, Moyano y Borzone, 2011). La ortografía debiera llegar a automatizarse, lo cual permitiría utilizar la memoria de trabajo en otros procesos (Pujol, 2000). Como han señalado Cuetos, Ramos y Ruano (2004), es posible escribir una palabra determinada sin necesidad de realizar ningún análisis intermedio, simplemente partiendo de la representación definida de la ortografía de la palabra, garantizada por un alto nivel de activación en el almacenaje de la misma, haciendo automática su correcta escritura, lo que han corroborado también García y González (2000).

El conocimiento léxico ortográfico que posea una persona se demuestra a la hora de escribir (Jiménez-Fernández, Defior y Serrano, 2010) por medio de la frecuencia y la lexicalidad: cuanto más frecuente y reconocible sea una palabra, más correctamente se escribirá. Igualmente, el nivel de vocabulario está estrechamente vinculado con el dominio ortográfico. Campbell, Yagelski y Yu (2014) han concluido que los educadores podrían reducir la frecuencia de errores de sus estudiantes si centraran su atención en los errores más comunes. Carratalá (2006) ha afirmado que las reglas ortográficas deben ser pocas, abarcar un amplio número de vocablos de uso, aplicarse sin excepciones y tener una formulación sencilla y fácil de retener. Gómez (2005) 
ha comentado que la enseñanza obligatoria y el bachillerato deberían proporcionar al alumnado la competencia lecto-escritora suficiente para continuar estudios posteriores o para desenvolverse en los ámbitos social o profesional sin dificultad alguna. Cree que es un error dar por 'aprendida' la ortografía en alumnado de bachillerato y universidad. Señala que el tratamiento de la ortografía en los niveles de enseñanza no obligatoria y superior debe suponer un ejercicio de reflexión individual de cada alumno, concretando su vocabulario cacográfico, identificando las causas de los errores que comete y escogiendo el recurso didáctico que mejor solución dé a sus problemas ortográficos. Es decir, "una ortografía basada en el análisis de los errores del vocabulario expresivo escrito". (p. 144).

Morales y Hernández (2004) han afirmado que "la producción deficiente de textos, desde el punto de vista gramatical, revela que no ha habido aprendizaje, que los estudiantes no han tomado conciencia sobre la normativa ortográfica del español" (2004: 9). Consideran que, si los estudiantes ingresan a la universidad con deficiencias en relación con su competencia comunicativa, la universidad está en la obligación de crear situaciones significativas de aprendizaje para que estos estudiantes se hagan usuarios competentes y autónomos de la lengua escrita. Para Gómez (2008: 25) "la ortografía en la universidad es, sobre todo, un problema de actitud: el deseo de escribir sin faltas los textos y el vocabulario expresivo que se utilizan en ejercicios reales de comunicación". Arrieta y Meza (2000) consideran que no corresponde al sistema de Educación Superior la tarea de enseñar a los estudiantes a leer y a escribir, aunque los datos obtenidos en sus investigaciones demuestran lo contrario.

Por último, las actitudes adoptadas por el profesorado universitario acerca de esta problemática han sido analizada por Dimángano y Delgrosso (2016). Se han centrado en reflexionar acerca del lenguaje y la ortografía que poseen los alumnos universitarios y en caracterizar la opinión y actitud del docente acerca de la ortografía de sus estudiantes desde su propia experiencia ortográfica.

\section{Los aportes de la investigación}

Durante el curso académico 2015-2016 desarrollamos un Proyecto de Innovación Docente para el análisis ortocaligráfico del alumnado universitario de los Grados de Maestro en la Facultad de Educación de la Universidad de Extremadura. La investigación consistió en la aplicación de cuestionarios y en el análisis de textos escritos de carácter académico elaborados por el alumnado (exámenes, trabajos, correos electrónicos e intervenciones en foros, relacionados con diversas asignaturas del plan de estudios). De ese amplio estudio, cuyos resultados se fueron concretando a lo largo del curso 2016-2017, presentamos ahora una parte. Se trata de un cuestionario ortográfico aplicado a 121 alumno del Grado de Maestro (65 de Infantil y 56 de Primaria) que cursaban $3^{\circ}$ y $2^{\circ}$ respectivamente y comprendidos entre los 19 y 22 años.

El instrumento de evaluación fue una prueba objetiva sobre ortografía, recogida de un manual de cultura general, empleado en el ámbito de las oposiciones a escalas básicas del funcionariado (ver Anexo Figura 1), prueba adaptada para ser superada al terminar la Educación Secundaria Obligatoria. El equipo investigador estimó su procedencia por cuanto evalúa aspectos ortográficos claves que suelen convertirse en los errores más habituales entre el ciudadano de a pie, pero menos probable entre el alumnado universitario, cuya formación precedente ha debido habilitarle mejor. 
El cuestionario estaba constituido por 40 palabras; pedía al alumnado universitario que indicara si estaban bien o mal escritas. Éramos conocedores que en el mismo se incluían palabras que exigían el conocimiento y dominio de la $\mathrm{H}(42,5 \%$ del total de palabras), la acentuación (32,5\%), el uso de Y/LL (5\%), la M antes de P (5\%), el uso de MN/NN (2,5\%), la utilización de S/X (10\%) o el empleo de G/J (2,5\%), representando porcentajes similares a los exigidos en la ortografía de palabras que constituyen el vocabulario fundamental del español.

Las pruebas, como la escogida, en las que se mide los conocimientos pasivos (lo que el alumnado debe saber, aunque no lo emplee en su comunicación habitual) nos parecen muy recomendables para el alumnado de los Grados de Maestro, puesto que en su ejercicio profesional se verán enfrentados a situaciones en las que deberán juzgar o evaluar producciones de sus alumnos y de otros adultos.

Según Mora (2007), el uso de las pruebas informales permite la detección de las habilidades de cada estudiante. Para Colorín (2007) las evaluaciones informales permiten a los docentes identificar las áreas de problemas específicos de los estudiantes, adaptar la enseñanza e intervenir en el momento indicado. Para Tejada (1997) la evaluación es un proceso sistemático de recogida de información, debe ir acompañada de la emisión de un juicio de valor y orientada a la toma de decisiones. De los tres tipos existentes (diagnóstica, formativa y sumativa), la diagnóstica tiene por objeto conocer la situación de partida. Desde el punto de vista del alumnado permite averiguar los conocimientos que poseen los alumnos con respecto a un asunto concreto. Aguilar-Morales (2011) ha afirmado que existen situaciones en las que el procedimiento de evaluación formal no es necesario ni aconsejable. Cuando son pocas las probabilidades de que el estudiante posea los conocimientos y habilidades que se le enseñan, es recomendable utilizar una evaluación informal, también recomendable cuando se pretende obtener información cualitativa del proceso educativo. La evaluación informal permite la emisión de un juicio sin que necesariamente se haya recabado la información a través de instrumentos de medición estandarizados y sin seguir un procedimiento determinado. De ahí nuestra elección.

La prueba se aplicó en sesiones diferentes a grupos reducidos de un máximo de 35 alumnos, aprovechando los seminarios prácticos programados para la asignatura de Didáctica de la Lengua. Eso permitió trabajar en un ambiente de concentración y no masificado. Durante un tiempo limitado y controlado (20 minutos) la prueba fue proyectada y mostrada en una pantalla con buena visibilidad. El profesor pronunciaba las palabras o leía los enunciados y las respectivas opciones en voz alta cada treinta segundos. El alumnado anotaba en un folio, dispuesto al efecto, las respuestas que creía adecuadas. Pasado el tiempo se interrumpió la proyección y se recogieron las hojas de respuestas. Las sesiones concluyeron con la corrección de la prueba por los propios alumnos, pero sin que les correspondiera la propia. Los resultados fueron comentados en un debate abierto que permitió concienciar al alumnado acerca de sus errores, con la finalidad de que pudieran ir elaborando su propio vocabulario cacográfico y reteniendo el tipo de error ortográfico más frecuente.

Del conjunto total del alumnado evaluado, el $75 \%$ respondió acertadamente, indicando qué palabras estaban bien o mal escritas; sin embargo, el 19\% erró en sus apreciaciones y un $6 \%$ prefirió dejar cuestiones sin responder. Estos porcentajes fueron muy similares en cada uno de los grupos por separado, ligeramente mejores en el grupo de Infantil, donde el 77\% anotó respuestas acertadas. Respecto al sexo, no 
podemos destacar representatividad, porque la muestra está muy descompensada a favor de las chicas. Por el contrario, aunque podría ser una variable válida, tampoco se pueden señalar diferencias significativas respecto al curso en que se encuentra el alumnado ( $3^{\circ}$ Infantil y $2^{\circ}$ Primaria), porque los resultados obtenidos son muy parecidos. El grupo de Infantil obtuvo mejores resultados a la hora de acertar las palabras de las preguntas $6,8,9,10,18,20,21,25,35,36$, consiguiendo el pleno acierto (65 sobre 65) en la palabra presentada en la pregunta 20 (Hormiga $=$ Bien), y casi pleno $(64$ de 65$)$ en las preguntas $6($ cohete $=$ Bien $)$ y $25($ colunna $=$ Mal $)$. Sin embargo, los peores resultados se obtuvieron en las preguntas 14,15 y 28, a la hora de determinar si estaban bien o mal escritas palabras como Ule = mal (14), alaja = mal (15) y tapíz $=$ mal (28). Palabras como ainco $=$ mal (5) o iergo $=$ mal (22) también registraron un elevado número de errores. Basta comprobar la ortografía de las palabras que más errores provocaron entre el alumnado para apreciar que la mayoría implicó el uso de la $\mathrm{H}$ y en menor medida la acentuación.

Por su parte, el grupo de Primaria obtuvo mejores resultados, por número de aciertos, en las preguntas 4, 6, 8, 9, 10, 20, 21, 24, 26, 27, 34 y 36, consiguiendo varios plenos (56 de 56) en las palabra presentadas en las preguntas 9, 20 y 21 (igue$\mathrm{ra}=$ mal, hormiga $=$ bien, ierro $=$ mal, respectivamente $)$, y casi pleno $(55$ de 56$)$ en la palabra de la pregunta 8 (proibir $=$ mal). Los peores resultados, sin embargo, se obtuvieron en las preguntas 14, 15, 19, 28 y 29. En estas preguntas las palabras más erradas coinciden con las equivocadas por el alumnado de Infantil, más irsuto = mal (19) y extreñido $=$ mal (29). Además, palabras como ache $=$ mal (12), emiplejía $=$ mal (16), orchata $=$ mal (17), ospedar $=$ mal (18), extratagema $=$ mal $(30)$, escelso = mal (32), exención = bien (33) y decágramo = mal (40) también registran un elevado número de alumnos que las señalan erróneamente. En este caso el resumen de errores es más amplio. Aparte de la $\mathrm{H}$, nos encontramos con descuidos en el uso de la S/X y la acentuación.

Comprobamos, pues, que el alumnado de Primaria, aparte de cometer un mayor número de errores ortográficos (21\% frente al 18\% de Infantil) los diversifica más. El sistema individualizado de evaluación nos permitió implicar al alumnado en su propio proceso de reflexión y corrección ortográfica. En este sentido, nos llamó mucho la atención que en el grupo de Educación Infantil tres chicas cometieran un número de errores significativamente importante. En dos casos estaban justificados, pues se trató de alumnado extranjero con intercambio Erasmus, pero en el tercero se trató de una alumna nativa de español que cometió 22 errores en las cuarenta palabras presentadas. Sorprendió que esta alumna pudiera 'desconocer' y equivocar la grafía correcta de: osar = bien (1); hóseo = mal (2); ovalo = mal (3); hipodromo $=$ mal (4); emisferio $=$ mal (11); emiplejía $=$ mal (16); orchata = mal (17); ospedar $=$ mal (18); irsuto $=$ mal (19); adiacente $=$ mal (23); album $=$ mal (26); extreñido $=$ mal (29); extratagema $=$ mal (30); pléiade $=$ mal (31); escelso $=$ mal (32); condor $=$ mal (39) y decágramo $=$ mal (40). Con esta serie de errores se ven implicadas reglas ortográficas, además de la acentuación, como la H, S/X, Y/LL. En el grupo de Primaria ningún alumno registró un número de errores significativos. Quien más errores cometió fue un chico con un total de 14, indicando desconocimiento o equivocación a la hora de determinar la ortografía correcta de: hóseo = mal (2); aínco $=$ mal (5); omenage $=$ mal (10); emisferio = mal (11); ache = mal (12); hangosto $=$ mal (13); ule = mal (14); alaja = mal (15); emiplejía = mal (16); orchata = mal (17); irsuto $=$ mal (19); iergo $=$ mal (22); tapíz $=$ mal (28); extratagema $=$ mal (30). Esta 
serie de errores implica a reglas ortográficas como la acentuación, el uso de H, G/J, $\mathrm{Y} / \mathrm{LL}, \mathrm{S} / \mathrm{X}$.

El cuestionario que hemos aplicado solo ha pretendido conocer el nivel de dominio ortográfico pasivo del alumnado universitario, para corroborar lo que ya han demostrados otros estudios: la elevada incidencia que esta disrupción normativa referida al ámbito comunicativo sigue teniendo en el contexto de la educación superior. No obstante, si lo hubiéramos utilizado como prueba tipo test para calificar el nivel de dominio ortográfico, como si de un examen se tratara, descontado los errores de los aciertos para eliminar el azar (práctica habitual en pruebas de este tipo), los resultados hubieran sido peores. Finalmente, y referido al estudio en general, el hecho de que las reglas ortográficas en las que se equivoca el alumnado sean acertadas unas veces y otras no, confirma la falta de atención, desinterés o descuido y, sobre todo, evidentes debilidades en el vocabulario. Esto quedó corroborado por los gestos de extrañeza de muchos universitarios al oír algunas palabras.

\section{Conclusiones y discusión de los resultados}

La evaluación llevada a cabo y la metodología que hemos seguido coinciden con lo que muy acertadamente han señalado Díaz y Manjón (2010): dar importancia a la caracterización de las dificultades ortográficas y guiar al alumnado para que pueda descubrirlas. Sánchez (2010) ha afirmado que los errores se pueden abordar con distintas estrategias, como la revisión y edición en grupos, el intercambio de escritos, la corrección colaborativa o el registro de las dificultades más frecuentes. Los estudios llevados a cabo por Atorresi et al. (2010), así como los desarrollados por Tuana (1980); Tuana, Carbonell y Luch, (1980) entre otros, indican que existen dificultades ortográficas vinculadas al uso de la tilde y que las palabras de uso común son las que se escriben con mayor frecuencia de manera incorrecta. Asimismo, se constata que las mujeres tienen un mejor desempeño ortográfico que los hombres. Los errores cometidos por los universitarios se centran, sobre todo, en el empleo de palabras aparentemente frecuentes en su vocabulario común y básico. Igualmente, el grupo de Infantil, en el que predomina el alumnado femenino, obtiene en su conjunto mejores resultados que el de Primaria.

Similares resultados encontraron Backhoff, Peon, Andrade y Rivera (2008). Evaluaron 27 tipos de errores ortográficos y el más frecuente fue el de acentuación, que se presentaba en casi la totalidad de los alumnos. Al analizar este problema, según la variable de sexo, se encontró que las mujeres presentaban un mejor desempeño. Otros problemas ortográficos frecuentes encontrados en su estudio fueron el uso de B/V, el uso de mayúsculas y el uso de S/C/Z. Bedwell, Domínguez, Sotomayor, Gómez y Jéldrez (2014) demostraron que cerca de la mitad de los errores que comente el alumnado corresponde a carencia de tildes. También se encontraron errores relacionados con la omisión de letras y sílabas, el uso erróneo de grafías $(\mathrm{B} / \mathrm{V}, \mathrm{H}, \mathrm{S} / \mathrm{C} / \mathrm{Z})$ y la hiposegmentación. En concreto, el uso erróneo de esas grafías corresponde al $21 \%$ del total de errores. Carratalá (2006) ya consideró como causas que, a su juicio, provocan las faltas de ortografía la escasa práctica lectora, contenidos relacionados con la enseñanza de la ortografía totalmente inadecuados, métodos basados en los criterios correctivos y poco razonables, entre otros. Entre sus propuestas didácticas mencionaba hacer que el alumnado interiorice las reglas mediante estrategias visoaudio-motor-gnósicas, elaborando un inventario. 
Backhoff et al., (2008) y Tuana (1980) han insistido en que la enseñanza de la ortografía debe enfocarse más en el análisis y reflexión sobre los errores ortográficos que en el aprendizaje de normas. Lo mismo han señalado Sotomayor, Molina, Bedwell, y Hernández (2013); Molina (2012) o Atorresi et al. (2010), destacando que los errores se presentan en mayor medida en el vocabulario de uso frecuente de los niños y jóvenes. Morales y Hernández (2004) han concluido que hay desconocimiento del sistema ortográfico y, por tanto, no se usa apropiadamente. Igualmente, el uso de las convenciones ortográficas de los estudiantes universitarios de nuevo ingreso no está determinado por las condiciones socioeconómicas y la formación profesional de los padres, ni por el tipo de educación que recibieron, sino en la concepción de su educación previa; en el desinterés por la lengua escrita que la escuela y la familia han desarrollado en el niño y, en consecuencia, en el joven y el adulto. Gómez (2008) propuso a su alumnado la elaboración de inventarios cacográficos personales en los que se recogieran los errores cometidos en situaciones reales de comunicación durante un cuatrimestre académico. Después, incluyó la necesaria reflexión sobre el origen del error.

Las conclusiones a las que nos ha conducido nuestro estudio van por esta línea: la irregularidad mostrada por el alumnado universitario a la hora de señalar errores ortográficos en series de palabras, acertándolas unas veces y errándolas otras, refuerzan la percepción de que conocen las reglas, pero su falta de atención, el desinterés por sus propios aprendizajes, el reducido vocabulario como consecuencia de hábitos lectores deficitarios y cierta crisis generalizada sobre valores, perspectivas de futuro, utilidad de la formación que reciben, etc. se proyecta sobre sus prácticas escritoras y sus usos ortográficos. Las escasas diferencias observadas en la variable del curso académico en que se encuentra matriculado el alumnado confirma que la realidad se mantiene en el tiempo, sin mejora alguna como consecuencia del proceso madurativo o de aprendizaje.

A diferencia de otros estudios, el alumnado analizado focaliza sus dificultades ortográficas sobre el uso de la $\mathrm{H}$, la acentuación, el uso de $\mathrm{S} / \mathrm{X}$ y el de $\mathrm{G} / \mathrm{J}$, que no aparecían en otros estudios similares. En ellos se ponen en evidencia no un conocimiento de unas determinadas reglas, sino lo que se conoce como la ortografía natural o fonética, que se manifiesta de manera más evidente en los primeros cursos de la educación básica, cuando el alumno está iniciando el proceso lecto-escritor. Algo impensable en el ámbito de la educación superior con alumnos que acumulan un largo proceso formativo, un amplio currículum académico.

Cuando se favorece la reflexión, el interés, la atención y se refuerza la importancia que tiene la comunicación para las relaciones interpersonales, especialmente en personas que centrarán su dedicación profesional en la formación de niños y jóvenes, los resultados mejorarán significativamente, porque el alumnado se conciencia del problema, presta mayor atención e interés y asume la responsabilidad que conlleva los estudios que está realizando. El propio alumnado universitario que participó en el estudio se asombró de los errores cometidos, reconoció no haber prestado la atención suficiente y demostró conocer las reglas en series complementarias que se utilizaron en la fase de reflexión.

Esta situación deberá ser tenida en cuenta por el profesorado universitario como parte de la formación global que recibe el alumnado y debe ser incorporada como mejora en los planes de estudio, por más que existan ciertas resistencias a admitir que el tema ortográfico pueda ser un asunto universitario, circunscrito hasta hace muy poco tiempo a los ámbitos de la educación primaria y secundaria. 


\section{Bibliografía}

Aguilar-Morales, Jorge Everardo (2011): La evaluación educativa. México: Asociación Oaxaqueña de Psicología A.C.

Alvar, Manuel (1979): Fonética, fonología y ortografía. LEA, 1/2, 211-231 (reimpreso en 1982). La lengua como libertad y otros estudios, Madrid: Ediciones Cultura Hispánica del Instituto de Cooperación Iberoamericana.

Arrieta de Meza, Beatriz y Meza Cepeda, Rafael (2000): Algunas estrategias para el mejoramiento de la lectura y de la redacción en estudiantes universitarios de recién ingreso. Revista Investigación y Postgrado. Vol. 15, nº.1, 291-311. Caracas: Universidad Pedagógica Experimental Libertador.

Atorresi, Ana et al, (2010): Escritura. Un estudio de las habilidades de los estudiantes de América Latina y el Caribe. Santiago: Unesco-Orealc.

Backhoff Escudero, Eduardo; Peon Zapata, Margarita; Andrade Muñoz, Edgar y Rivera LÓPez, Sara (2008): La ortografía de los estudiantes de educación básica en México. México DF: Instituto Nacional para la Evaluación de la Educación. [Disponible en http://www. oei.es/pdf2/ortografia_estudiantes_basica_mexico.pdf. Consultado el 21 de abril de 2017].

Bedwell, Percy; Domínguez, Ana María; Sotomayor, Carmen; Gómez, Gabriela y Jeldrez, Elvira (2014): Caracterización de Problemas Ortográficos Recurrentes en Alumnos de Cuarto Básico, en Alameda, José Ramón y Cuetos, Fernando (1995). Diccionario de frecuencias de las unidades lingüísticas del castellano. Oviedo: Servicio de Publicaciones de la Universidad.

Berninger, Virginia et al. (1992): Lower-level developmental skills in beginning writing. Reading and Writing, 4, 257-280.

Campbell, Kristen; Yagelski, Robert y Yu, Fang (2014): The nature of error in adolescent student writing. Reading and Writing, 27, 1073-1094.

Camps, Anna et al. (1990): La enseñanza de la ortografía. Barcelona: GRAO.

Carratalá, Fernando (2006): La enseñanza integrada de la ortografía y el vocabulario en la Enseñanza Secundaria. Material didáctico. Madrid: Comunidad de Madrid.

Colorín Colorado (2007): Usar evaluaciones informales para estudiantes. Estados Unidos. [Disponible en http://www.colorincolorado.org/educadores/evaluacion/informal/. Consultado el 4 de mayo de 2017]

Cuetos, Fernando; Ramos Sánchez, José Luis y Ruano Hernández, Elvira (2004): PROESC. Evaluación de los procesos de escritura. Madrid: Tea Ediciones.

Díaz Perea, María del Rosario (2008): Preocupaciones docentes y enfoque didáctico de la enseñanza de la ortografía. Docencia e Investigación: revista de la Escuela Universitaria de Magisterio de Toledo, $\mathrm{n}^{\circ}$. 18. [Disponible en: https://www.uclm.es/varios/revistas/docenciaeinvestigacion/pdf/numero8/Rosario_Diaz_Perea.doc. Consultado el 4 de abril de 2017].

Dimángano, Patricia y Delgrosso, Adriana (2016): La disortografía en universitarios: estudio descriptivo de la actitud y opinión de los docentes. Revista da UIIPS, no 4, Santarem: Instituto Politécnico.

García, Jesús y González, Daniel (2000): Dificultades del Aprendizaje e intervención psicopedagógica. Volumen II. Lectura y Escritura. Madrid: EOS.

Gomes de Moráis, Artur y Teberosky, Ana (1993): Escribir con o sin errores de ortografía. Cuadernos de Pedagogía, $\mathrm{n}^{\circ} 216,57-59$.

Gómez, Alejandro (2005): Enseñar ortografía a universitarios andaluces. En Escuela Abierta, $\mathrm{n}^{\mathrm{o}} 8,129-147$. 
Gómez Camacho, Alejandro (2008), La educación secundaria de Andalucía y la ortografía del español. Perspectiva CEP, n ${ }^{\circ} 13,109-132$.

Jiménez-Fernández, Gracia; Defior, Silvia Ana y Serrano, Francisca (2010): Adquisición de la escritura de palabras: modelos y metodología de estudio. En Carrillo, María Soledad y Domínguez, Ana Belén, Dislexia y Sordera. Líneas actuales en el estudio de la lengua escrita y sus dificultades. Málaga: Aljibe.

Matteoda, María Celia y Vázquez de Apra, Alicia (1990): Concepciones infantiles acerca del sistema ortográfico. Temas de Psicopedagogía, 4, 77-95.

Medina Guerra, Antonia María (1994): La enseñanza de la ortografía en la universidad en REALE, 2, 1994, 73-78.

Ministerio de educación, cultura y deporte (2002): Marco Europeo de referencia para las Lenguas: Aprendizaje, enseñanza, evaluación. Madrid: Subdirección General de Cooperación Internacional.

Molina Vargas, Daniela (2012): Habilidades de escritura en niños de educación básica: caracterización del desempeño ortográfico en narraciones de escolares de $3^{\circ}, 5^{\circ}$ y $7^{\circ}$ año de educación básica pertenecientes a escuelas municipales rurales de Constitución y Empedrado. [Disponible en http://www.repositorio.uchile.cl/handle/2250/115357. Consultado el 28 de marzo de 2017].

Mora Hernández, Lorena (2007): Evaluación Diagnóstica en la Atención de estudiantes con Necesidades Educativas Especiales. San José, Costa Rica: Editorial EUNED.

Morales, Óscar Alberto y Hernández, Luis (2004): Estudio descriptivo del uso de la ortografía de los estudiantes universitarios de nuevo ingreso. Kaleidoscopio, 2, 151-159.

Polo, José (1974): Ortografía y ciencia del lenguaje, Madrid: Paraninfo.

Pujol Llop, Mario (2000): Hacia una visión integrada de la ortografía: comunicativa, cognitiva y lingüística. Tabanque. Revista Pedagógica, nº 15, 223-248.

Salgado, Hugo (1997): Aprendizaje ortográfico en la didáctica de la escritura. Buenos Aires: Aique.

Sánchez, David (2010): El análisis de errores ortográficos de estudiantes filipinos en el aprendizaje de español como LE y su aplicación didáctica. I Congreso de Español como Lengua Extranjera en Asia-Pacífico, 106-126. [Disponible en http://cvc.cervantes.es/ensenanza/biblioteca_ele/publicaciones_centros/pdf/manila_2009/09_investigaciones_02. pdf. Consultado el 2 de abril de 2017].

Sánchez, Verónica; Moyano, Valeria y Borzone, Ana María (2011): Demandas cognitivas de la escritura: comparación de dos situaciones de producción. Estudios Pedagógicos, 37, 227-236.

Smith-Lock, Karen; Nickels, Lyndsey y Mortensen, Lynne (2009): Story writing skills of adults with a history language-impairment. Reading and Writing, $\mathrm{n}^{\circ} 22,713-734$.

Sotomayor, Carmen; Molina, Daniela; Bedwell, Percy y Hernández, Carolina (2013): Caracterización de problemas ortográficos recurrentes en alumnos de escuelas municipales chilenas de $3^{\circ}, 5^{\circ}$ y $7^{\circ}$ básico. Signos, 46 (81), 105-131. [Disponible en http://www.scielo.cl/ scielo.php?script $=$ sci_arttext\&pid=S0718-09342013000100005\&lng=es\&tlng=es. 10.4067/S0718-09342013000100005. Consultado el 28 de abril de 2017].

Tejada Fernández, José (1997): El proceso de investigación cientifica. Barcelona: E.U.I. Santa Madrona.

Tuana, Elida (1980). Estudio comparativo de la ortografía en niños normales y niños con dificultades de aprendizaje. Lectura y Vida, 1.

Tuana, Elida; Carbonell, María y Lluch, Elena (1980): Diez años de investigaciones ortográficas. Lectura y Vida, 2. 
Vaca, Jorge (1983): Ortografía y significado. Lectura y Vida, 4, (1), 4-9.

(1997): Lo no alfabético en el sistema de escritura: ¿Qué piensa el escolar?. México: CINVESTAV.

\section{Anexo}

\section{$0-13$}

Indique si cada palabra está escrita bien (B) 0 mal (M).

\begin{tabular}{|c|c|c|c|c|c|}
\hline 1. Osar & B & M & 2. & Hóseo & B \\
\hline Ovalo & B & M & 4. & Hipodromo & B \\
\hline Aínco & B & M & 6. & Cohete & B \\
\hline 7. Hulceroso & B & M & 8. & Proibir & B \\
\hline 9. Iguera & B & M & 10. & Omenage & $\mathrm{B}$ \\
\hline 11. Emisferio & $B$ & $M$ & 12. & Ache & B \\
\hline 13. Hangosto & B & $M$ & 14. & Ule & B \\
\hline 15. Alaja & B & M & 16. & Emiplejía & B \\
\hline 17. Orchata & B & M & 18. & Ospedar & B \\
\hline 19. Irsuto & $\mathrm{B}$ & M & 20. & Hormiga & B \\
\hline 21. Ierro & B & M & 22. & Iergo & B \\
\hline 23. Adiacente & B & M & 24. & Inbécil & B \\
\hline 25. Colunna & B & M & 26. & Album & $\mathrm{B}$ \\
\hline 27. Esperiencia & B & M & 28. & Tapíz & B \\
\hline 29. Extreñido & B & M & 30. & Extratagema & $\mathrm{B}$ \\
\hline 31. Pléiade & B & M & 32. & Escelso & B \\
\hline 33. Exención & B & M & 34. & Mastil & B \\
\hline 35. Perifrasis & B & M & 36. & Optimo & B \\
\hline 37. Intervalo & B & M & 38. & Buho & B \\
\hline 39. Condor & B & M & 40. & Decágramo & B \\
\hline
\end{tabular}

Figura 1. 